ISSN 1991- 8690

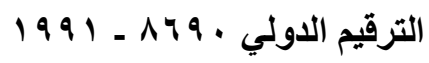

Website: http://jsci.utq.edu.iq

Email: utjsci@utq.edu.iq

تخليق و دراسات طيفية لبعض معقدات العناصر الانتقالية لقواعد شف المشتقة من المركبات ثنائية الأمين

ساهر عبد الرضا علي فاطمة فليح حسن

جامعة ذي قار _ كلية العلوم _قسم الكيمياء

الخلاصة

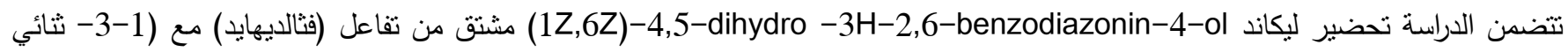

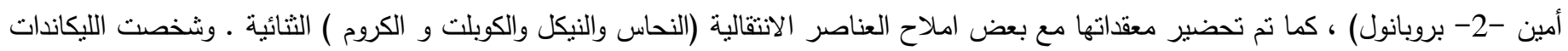

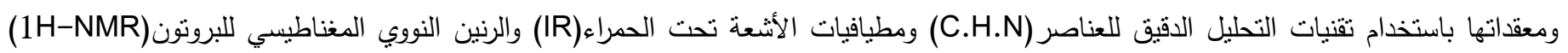

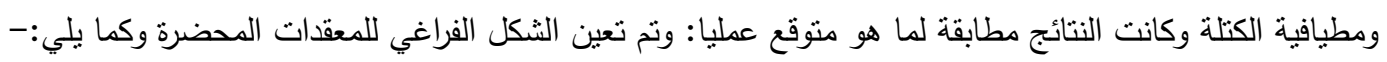
1- معقدات الكوبلت والكروم الثناثثة ثمانبة السطوح. 2- معقدات النيكل الثنائية رباعية السطوح.

الكلمات المفتاحية: معقدات قواعد شف

\title{
Synthesis, Spectroscopic Studies of Some Transition Metal Complexes of Schiff Bases Derived from Diamine Compounds
}

Fatima F. Hassan

Saher A. Ali

Thi-Qar University - College of Science

\section{$\underline{\text { Abstract }}$}

The present work include preparation of three ligands (Schiff base) by condensation of (Phthaldialdehyde) with (1,3-diamino-2-propanol) to give ligands (L)

L: (1Z,6Z)-4,5-dihydro -3H-2,6-benzodiazonin-4-ol

To characterize the structure of prepared ligands and their complexes, elemental analysis (C.H.N),Infrared Spectroscopy (IR), Nuclear Magnetic Resonance Spectroscopy $\left({ }^{1} \mathrm{H}-\mathrm{NMR}\right)$,Mass Spectra and Molar conductivity techniques were applied. The results showed that:

1- The complexes of [Co(III) and $\mathrm{Cr}(\mathrm{III})]$ for ligand have shown octahedral configuration.

2- Complexes of [ $\mathrm{Ni}(\mathrm{II})]$ with ligands have shown tetrahedral configuration.

Keywords: Schiff base complexes

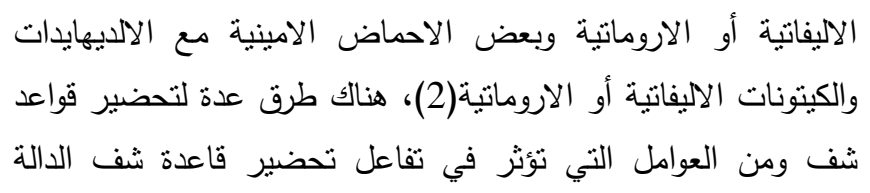

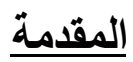

حُضرت قواعد شف لأول مرة من قبل العالم الألماني هوكو شف عام (Hugo Schiff) 


\section{(2) تحضير معقدات العناصر الأنتقالية:}

تم استخدام الطريقة العامة الآتية في تحضير المعقدات مع الليكاند

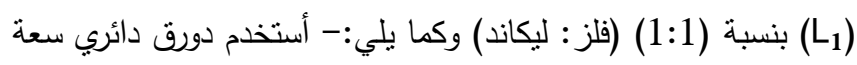

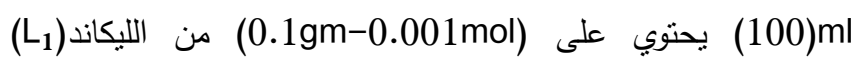

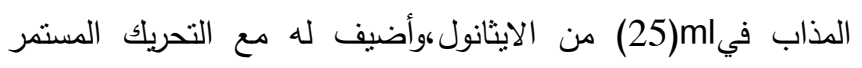

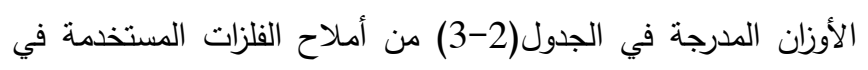

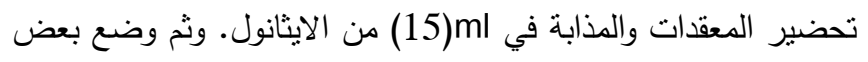

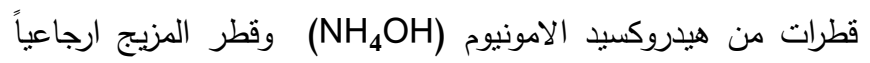

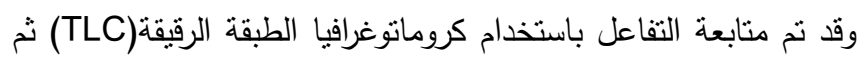

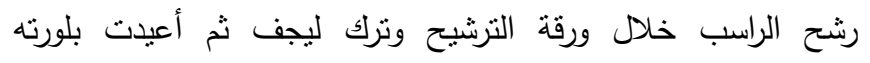

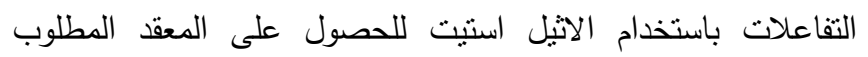

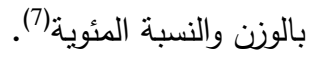

جدول (1): الصيغة الجزيئية وبعض الخواص الفيزيائية لمعقدات L1

\begin{tabular}{|c|c|c|c|c|c|}
\hline المرقي. & الصيفة الجزيئية & الهونغ الجزئي & الآلهوان & الرجات الآصهار(C) & الحصيأة) (\%) \\
\hline 1 & $\mathrm{C}_{11} \mathrm{H}_{12} \mathrm{~N}_{2} \mathrm{O}$ & 188 & بني غامق & (179-181) & 73 \\
\hline 2 & {$\left[\mathrm{CO}\left(\mathrm{L}_{1}\right)_{2} \mathrm{C}_{\mathrm{l}}\right] \mathrm{Cl}$} & 540 & أسود & $(221-222)$ & 68 \\
\hline 3 & $\|\left[\operatorname{Cr}\left(\mathrm{L}_{1}\right)_{2} \mathrm{C}_{\mathrm{l} 2}\right] \mathrm{Cl}$ & 533 & اصفر فتاتح & (126-129) & 67 \\
\hline 4 & {$\left[\mathrm{Ni}\left(\mathrm{L}_{1}\right)\left(\mathbf{S O}_{4}\right)\right]$} & 342 & 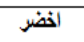 & $\begin{array}{l}(262-263) \\
\end{array}$ & 65 \\
\hline
\end{tabular}

النتائج والمناقشة:

(1) - (1) التحليل الاقيق للعناصر

الجدول (2) يمثل النسب العملية والنظرية التي تم الحصول عليها لليكاند المضر

\begin{tabular}{|c|c|c|c|c|c|c|}
\hline المركب & \multicolumn{3}{|c|}{ العطلي } & \multicolumn{3}{|c|}{ النتزري } \\
\hline \multirow[t]{2}{*}{$\mathrm{C}_{11} \mathrm{H}_{12} \mathrm{~N}_{2} \mathrm{O}$} & $\mathbf{N} \%$ & H\% & C \% & $\mathrm{N} \%$ & $\mathrm{H} \%$ & $\mathrm{C} \%$ \\
\hline & 14.926 & 6.55 & 70.265 & 14.88 & 6.43 & 70.19 \\
\hline
\end{tabular}

الحامضية و التأثيرات الالكترونية والفراغية لمركب الكاربونيل والأمين

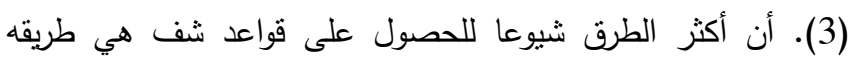

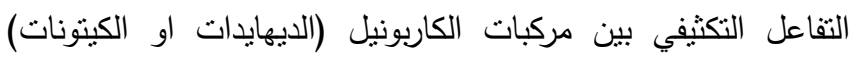
الاليفاتية أو الاروماتية مع الأمينات الاولية (الاليفاتية آو الاروماتية)

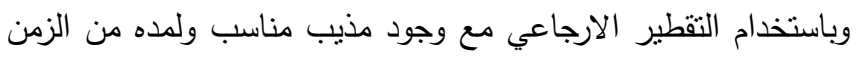
واكتثفت لاول مره من قبل العالم شف وسميت القواعد الناتجة بقواعد مند

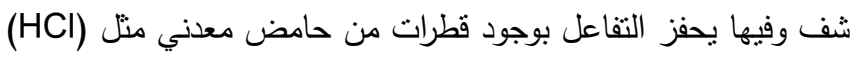
او حامض عضوي مثل (CH3COOH) وغالبا مايحصل الثناعل

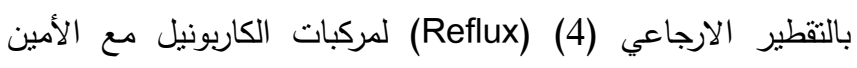

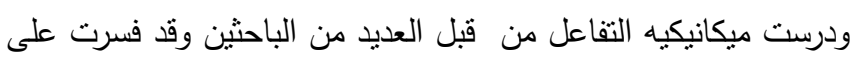
ان الحامض يهب بروتون لمجموعة الكاربونيل لنكوين ايون الكاربونيوم

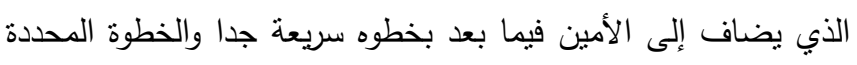

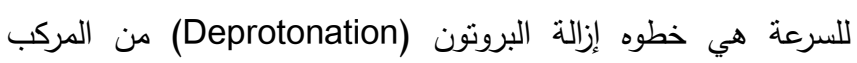
الوسطي لنكوين الكارينول أمين والذي يكون غير مستقر سرعان ما لإنا

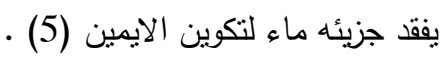
طريقة العمل: تحضــير المركسبات: (1) (1) تحضير الليكاند: (1Z,6Z)-4,5-dihydro -3H-2,6-benzodiazonin-4-ol

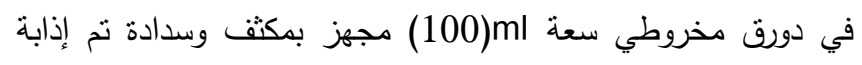

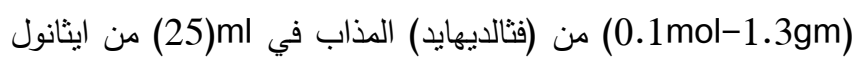

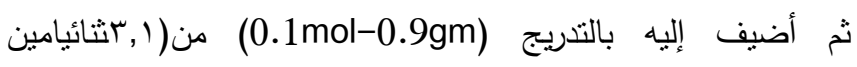

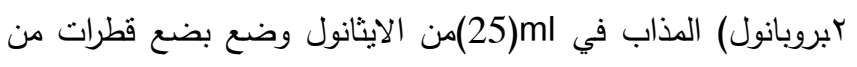

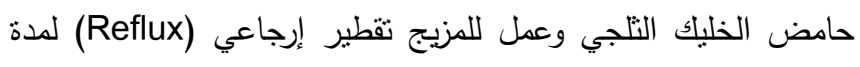

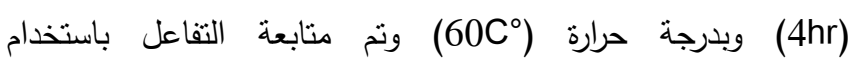

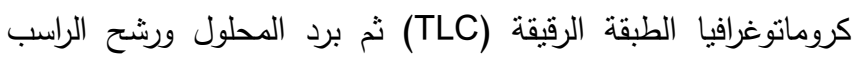
خلال ورقة الترشيح وترك ليجف ثم أعبد تبلور باستخدام الاينانول

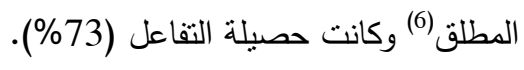

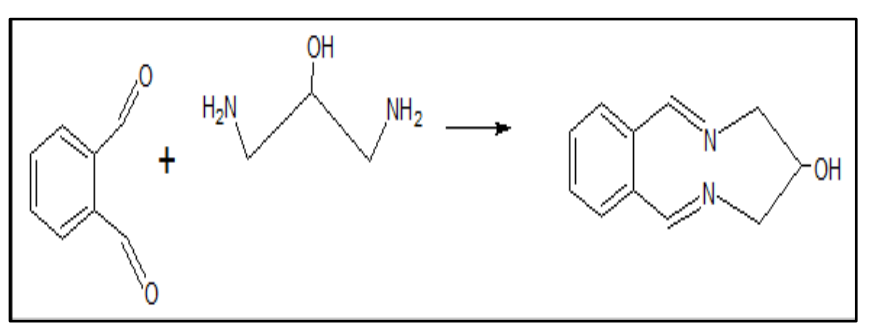




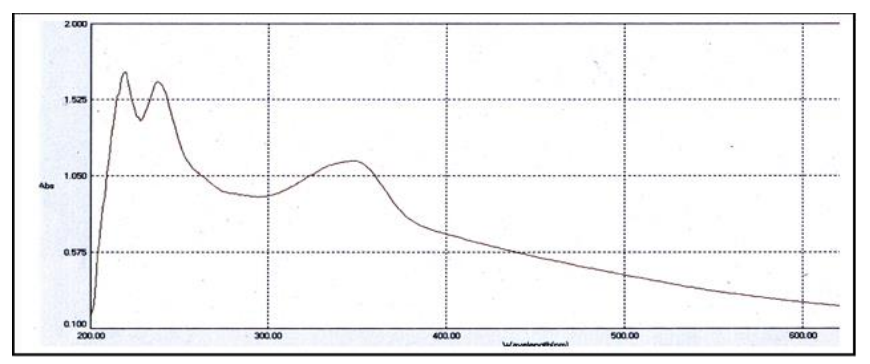

شكل (2): طيف الأشعة المرئية-فوق البنفسجية للمعقد [Co(L1)2Cl2]Cl

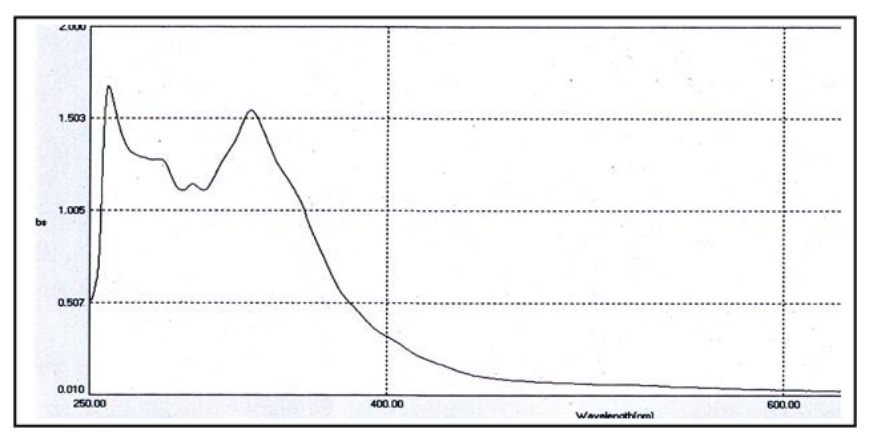

شكل (3): طيف الأشعةالمرئية- فوق البنفسجية للمعقد [Cr(L1)2Cl2]Cl

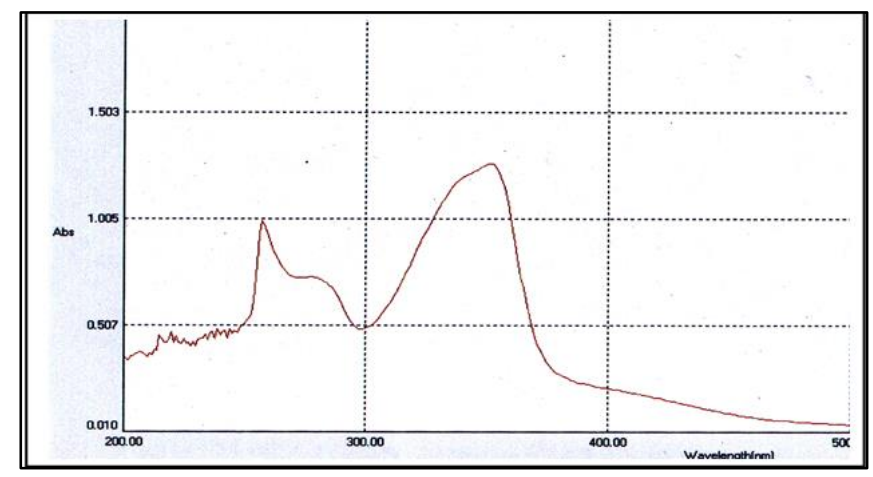

شكل (4): طيف الأشعةالمرئية-فوق البنفسجية للمعقد [Ni(

(2) الأطياف الالكترونية لليكاند المحضر ومعقداته: شُخص اليكاند المحضر ومعقداته بواسطة الاشعة فوق البنفسجية

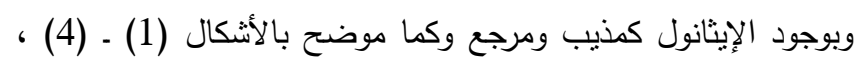

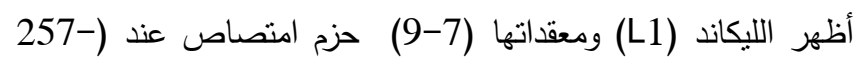

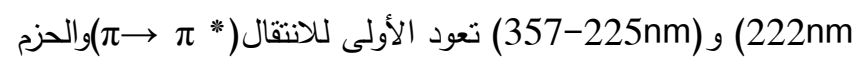
الأخرى للانتقال( البنفسجية للمعقدات المحضرة حزم امتصاص تنزاوح بين (-558

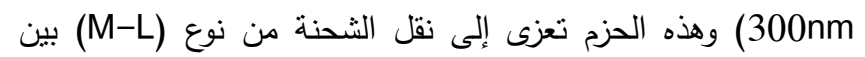

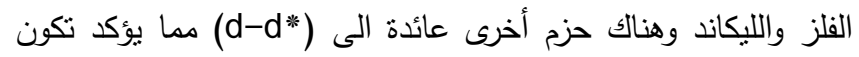

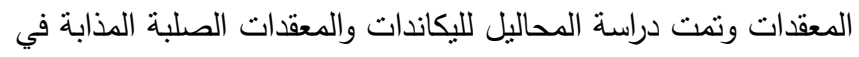

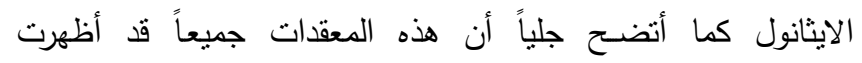

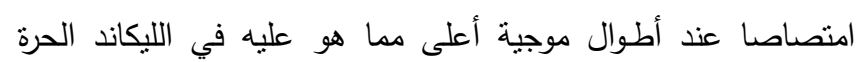

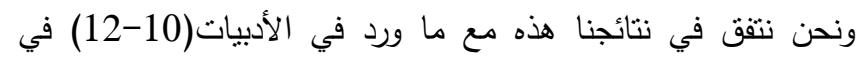

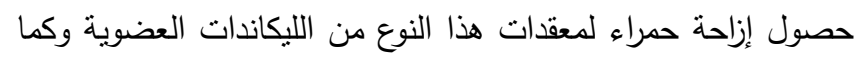

هي مبينة بالجدول(3).

جدول (3) الاطوال الموجية وانواع الانتقالات لليكاند المحضر

$$
\text { ومعقداته في الطيف الالكتروني }
$$

\begin{tabular}{|c|c|c|c|c|}
\hline 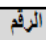 & الهركبات & الطول الموجي الأعظى & الإتقالات الالكترونية & الشكل الهندسي \\
\hline 1 & $\mathrm{C}_{11} \mathrm{H}_{12} \mathrm{~N}_{2} \mathrm{O}$ & $357,257,225,222$ & $\pi \rightarrow \pi^{*}, \mathrm{n} \rightarrow \pi^{*}$ & \\
\hline 2 & {$\left[\mathrm{Co}\left(\mathrm{L}_{2}\right)_{2} \mathrm{Cl}_{2}\right] \mathrm{Cl}$} & $347,238,220,296$ & $\pi \rightarrow \pi^{*}, \mathrm{~d} \rightarrow \mathrm{d}^{*}, \mathrm{n} \rightarrow \pi^{*}$ & ليدائي السطوح \\
\hline 3 & {$\left[\mathrm{Cr}\left(\mathrm{L}_{2}\right)_{2} \mathrm{Cl}_{2}\right] \mathrm{Cl}$} & $301,259,300,214$ & $\pi \rightarrow \pi^{*}, \mathrm{M} \rightarrow \mathrm{L}, \mathrm{n} \rightarrow \pi^{*}$ & أثدائي السطوح \\
\hline 4 & {$\left[\mathrm{Ni}\left(\mathrm{L}_{2}\right)\left(\mathrm{SO}_{4}\right)\right]$} & $492,360,350,238$ & $\pi \rightarrow \pi^{*}, \mathrm{M} \rightarrow \mathrm{L}, \mathrm{n} \rightarrow \pi^{*}$ & رباعي السطوح \\
\hline
\end{tabular}

وفيما يلي أثنكال طيف الأشعة المرئية - فوق البنفجية لليكاندات المحضرة ومعقداتها

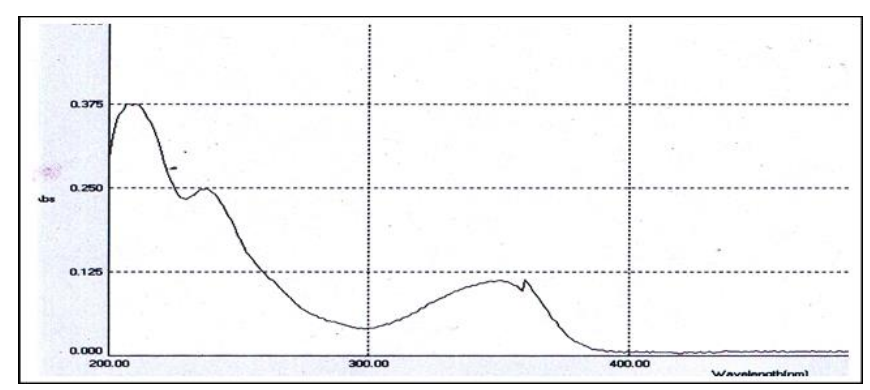

شكل (1): طيف الأشعةالمرئية-فوق البنفسجية لليكاند (C11H12N2O) 


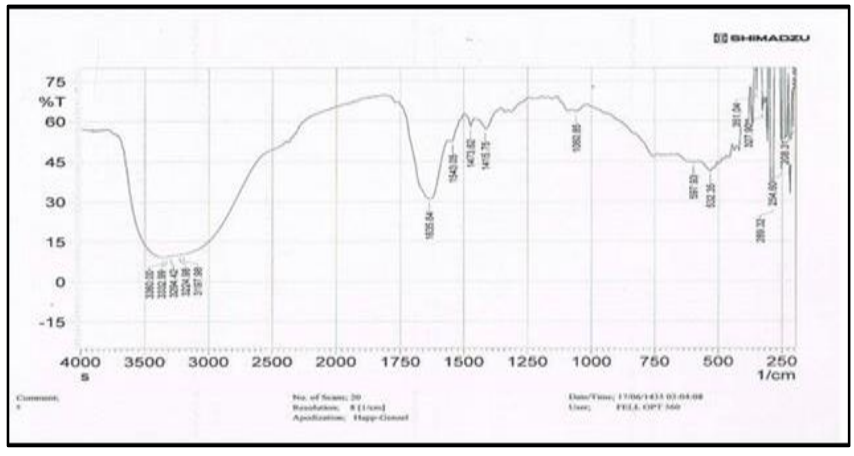

شكل (3): طيف الأشعة تحت الحمراء للمعقد

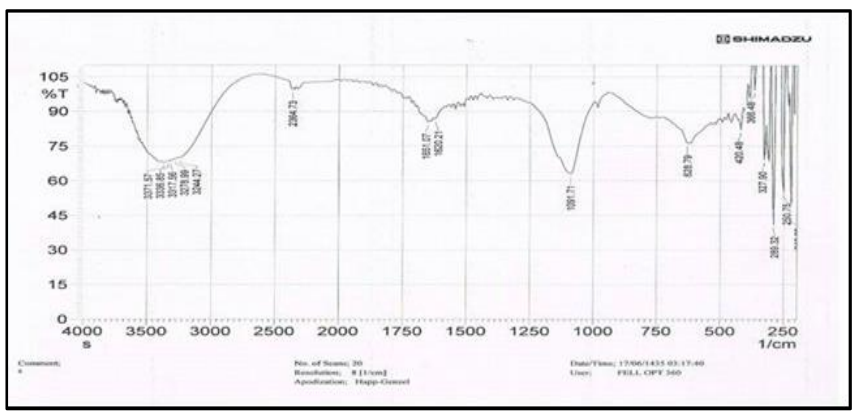

شكل (4): طيف الأشعة تحت الحمراء للمعقد [Ni(L1)(SO4)

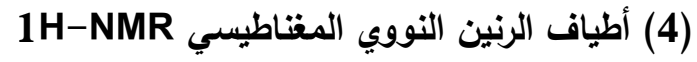

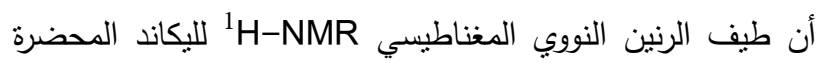

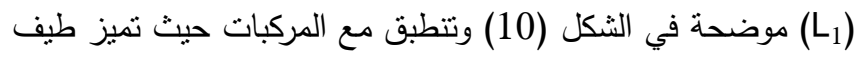

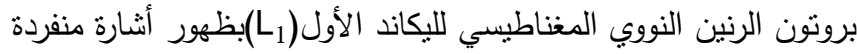

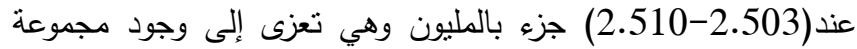
(CH2)

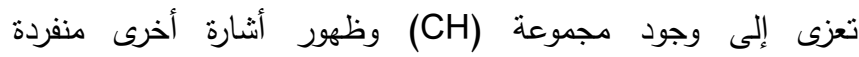
عند (5.783)جزء بالمليون وهي تعزى إلى وجود مجموعة الهيدروكسيل

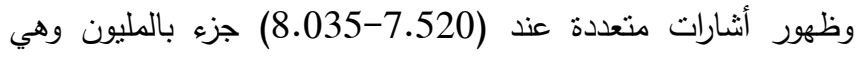
تعزى إلى بروتونات الحلقة الاورماتية (13-14) وظهور أثشارةعند

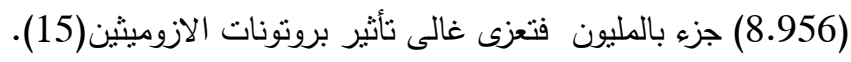

(3) أطياف الأثثعة تحت الحمراء

\begin{tabular}{|c|c|c|c|c|c|c|c|c|c|c|}
\hline \multicolumn{11}{|c|}{ الحمراءلليكاند جدول (4): حزم امتصاص المنطقة تحت $\mathrm{C}_{11} \mathrm{H}_{12} \mathrm{~N}_{2} \mathrm{O}$ ومعقداتها المحضرة بوحدا } \\
\hline المركبات & O-H & $\mathrm{C}-\mathrm{H}_{\mathrm{Ar} .}$ & $\mathrm{C}-\mathrm{H}_{\mathrm{AI}}$ & $\mathrm{C}=\mathrm{N}$ & $\mathrm{C}=\mathrm{C}$ & $\begin{array}{l}\text { C-O } \\
\text { C-N }\end{array}$ & $\begin{array}{c}\mathrm{C}-\mathrm{O} \\
\mathrm{C}-\mathrm{N}\end{array}$ & M-N & M-O & $\mathrm{M}-\mathrm{Cl}$ \\
\hline $\mathrm{C}_{11} \mathrm{H}_{12 \mathrm{~N}} \mathrm{O}$ & 3429 & 3050 & $\begin{array}{l}2920 \\
2850\end{array}$ & 1670 & 1473 & $\begin{array}{l}1419 \\
1319\end{array}$ & $\begin{array}{l}1087 \\
1064\end{array}$ & $=$ & - & \\
\hline$\left[\mathrm{Co}\left(\mathrm{L}_{2}\right)_{2} \mathrm{Cl} l_{2}\right] \mathrm{Cl}$ & \begin{tabular}{|l|}
3429 \\
3375 \\
\end{tabular} & 3064 & \begin{tabular}{|l|}
2939 \\
2839 \\
\end{tabular} & 1624 & 1591 & 1473 & 1070 & 667 & 420 & 254 \\
\hline$\left[\mathrm{Cr}\left(\mathrm{L}_{1}\right)_{2} \mathrm{Cl}\right] \mathrm{Cl}$ & \begin{tabular}{|l|}
3360 \\
3332 \\
\end{tabular} & 3059 & \begin{tabular}{|l|}
2931 \\
2850 \\
\end{tabular} & 1635 & 1543 & 1473 & 1060 & 597 & 532 & 289 \\
\hline$\left[\mathrm{Ni}\left(\mathrm{L}_{2}\right)\left(\mathrm{SO}_{4}\right)\right]$ & $\begin{array}{l}3371 \\
3336 \\
3317\end{array}$ & 3061 & 2841 & 1651 & 1550 & $\begin{array}{l}1438 \\
1375\end{array}$ & $\begin{array}{l}1091 \\
1012\end{array}$ & 628 & 420 & - \\
\hline
\end{tabular}

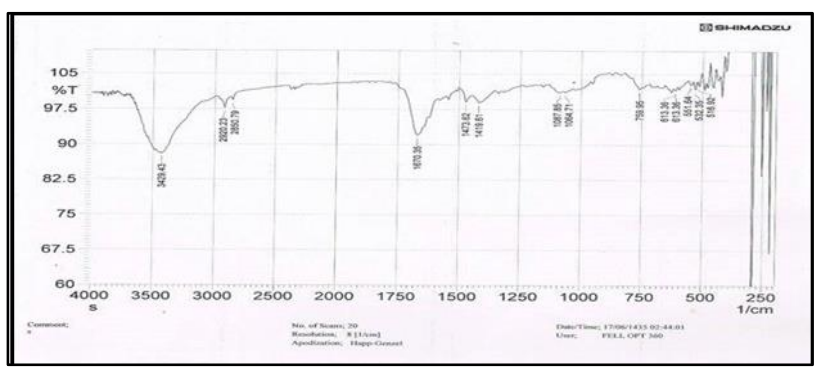

شكل (1): طيف الأشعة تحت الحمراء لليكاند (C11H12N2O)

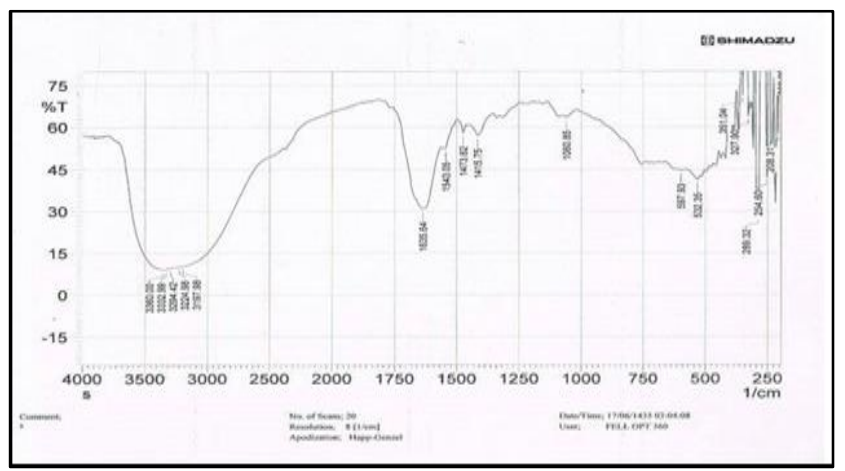

شكل (2): طيف الأشعة تحت الحمراء للمعقد 


\section{3- 3يف الكتلة للمعقد}

اظهر طيف الكتلة للمعقد الايون الجزيئي (M+.)

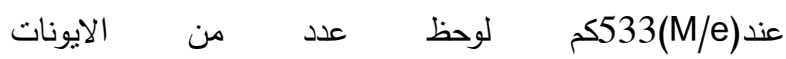

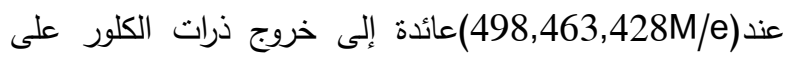

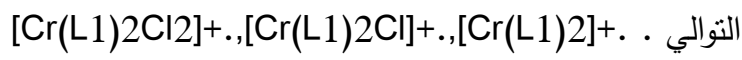

4- طيف الكتلة للمعقد [Ni(L1)(SO)4

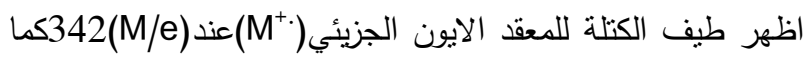
لوحظ ايون عند246(M/e(عائد إلى خروج جزيئة الكبرينات (Ni)+.

$\left.\left(L_{1}\right)\right]$

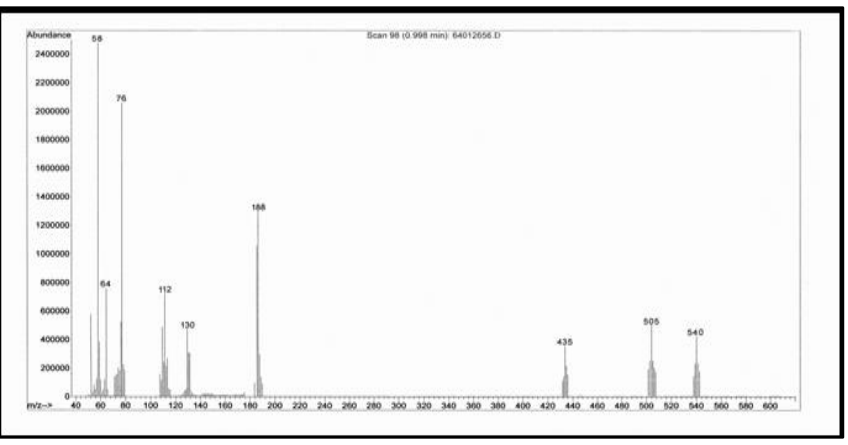

شكل (6): طيف الكتلة لليكاند (C11H12N2O)

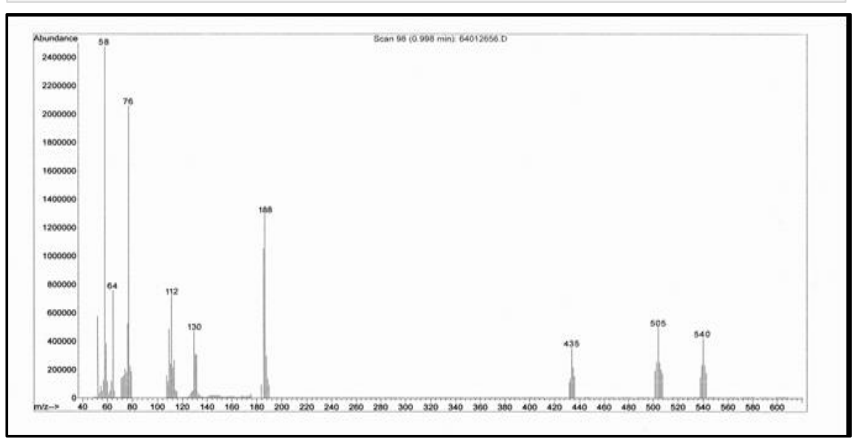

شكل (7): طيف الكتلة للمعقد

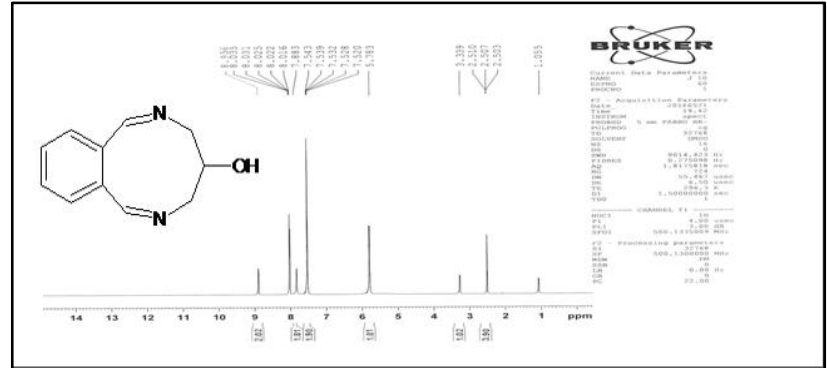

شكل (5): طيف الرنين النووي المغناطيسي لليكاند (C11H12N2O)

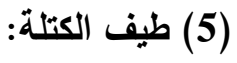
1 - طيف الكتلة لليكاند:

تميز طيف الكنلة الليكاند الثاني بظهور ذرة الايون الجزيئي(M

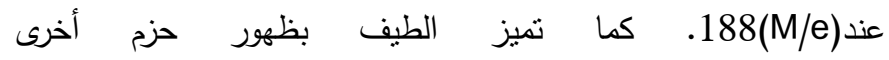
(M/e)130,112,76,64,58,44 تعود إلى نيز $\left[\mathrm{C}_{6} \mathrm{H}_{4}\right] .+,\left[\mathrm{C}_{5} \mathrm{H}_{4}\right] .+,\left[\mathrm{C}_{3} \mathrm{H}_{6} \mathrm{O}\right] .+,\left[\mathrm{C}_{2} \mathrm{H}_{4} \mathbf{0}\right] .+\left[\mathrm{C}_{8} \mathrm{H}_{6} \mathrm{~N}_{2}\right] .+,\left[\mathrm{C}_{5}\right.$ $\left.\mathrm{H}_{8} \mathrm{~N}_{2} \mathrm{O}\right]$.+

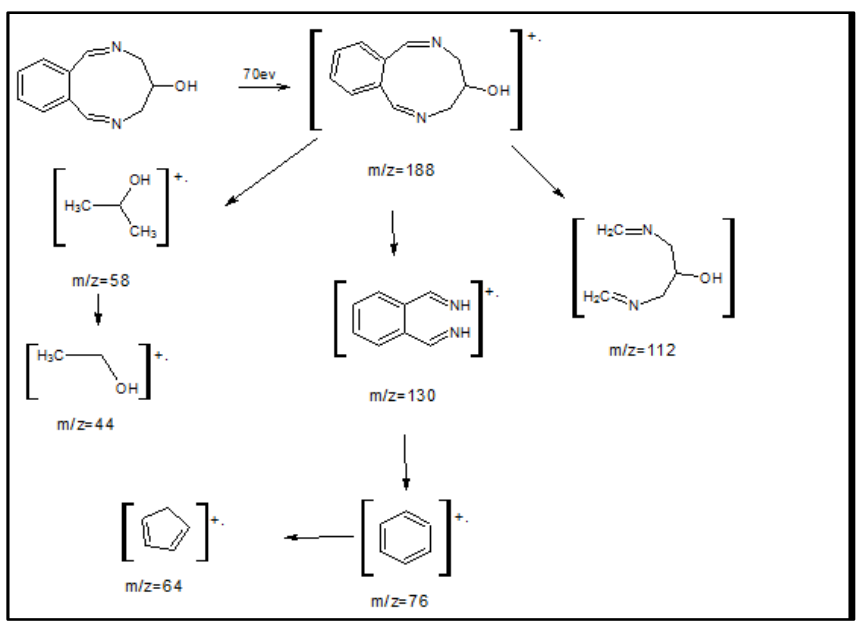

مسار أنشطارات طيف الكتلة لليكاند (L)

2- طيف الكتلة للمعق

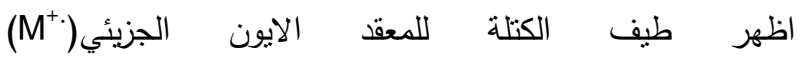

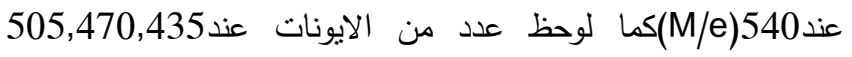

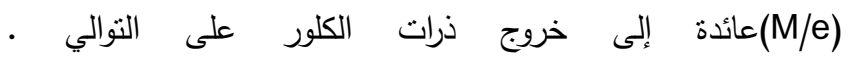
$\left[\mathrm{Co}\left(\mathrm{L}_{1}\right)_{2} \mathrm{Cl}_{2}\right]^{+\cdot}\left[\mathrm{Co}\left(\mathrm{L}_{1}\right)_{2} \mathrm{Cl}\right]^{+},\left[\mathrm{Co}\left(\mathrm{L}_{1}\right)_{2}\right]^{+}$ 
9- E. Ispir, S. Torog lu , A. Kayraldız,"Syntheses, characterization, antimicrobial and genotoxic activitiesof new Schiff bases and their complexes", Vol 10, pp 953-960,(2008).

10 - H.A.M.Saleh, A.A.Al-Kad Humi and N.A.Fakhri ; The Journal of The College of Education, Salahaddin University, Vol 2, pp 124,(1990).

11- V.Michaylova , B.Evtmova and D.Nonova ; Anal.Chem.Acta., Vol 207, pp 373,(1988).

12- Khalid J.K.Al-Adely, Ph .D.Thesis University of Baghdad, (2000).

13- J.F.M.dasilva,S.J.Garden,A.C.Pinto and J.Braz,Chem.Soc, Vol 12, pp 1-105,(2001).

14- A. Huang, J.J.Kodanko, L.E.Overman and J.Am. Chem,Sco, Vol 126, pp 14043-4053,(2004).

15- R.R. Kamble, B.S. Sudha and D.G. Bhadregowda, J.Serb, Chem.Soc, Vol 73, pp 131-138,(2008).
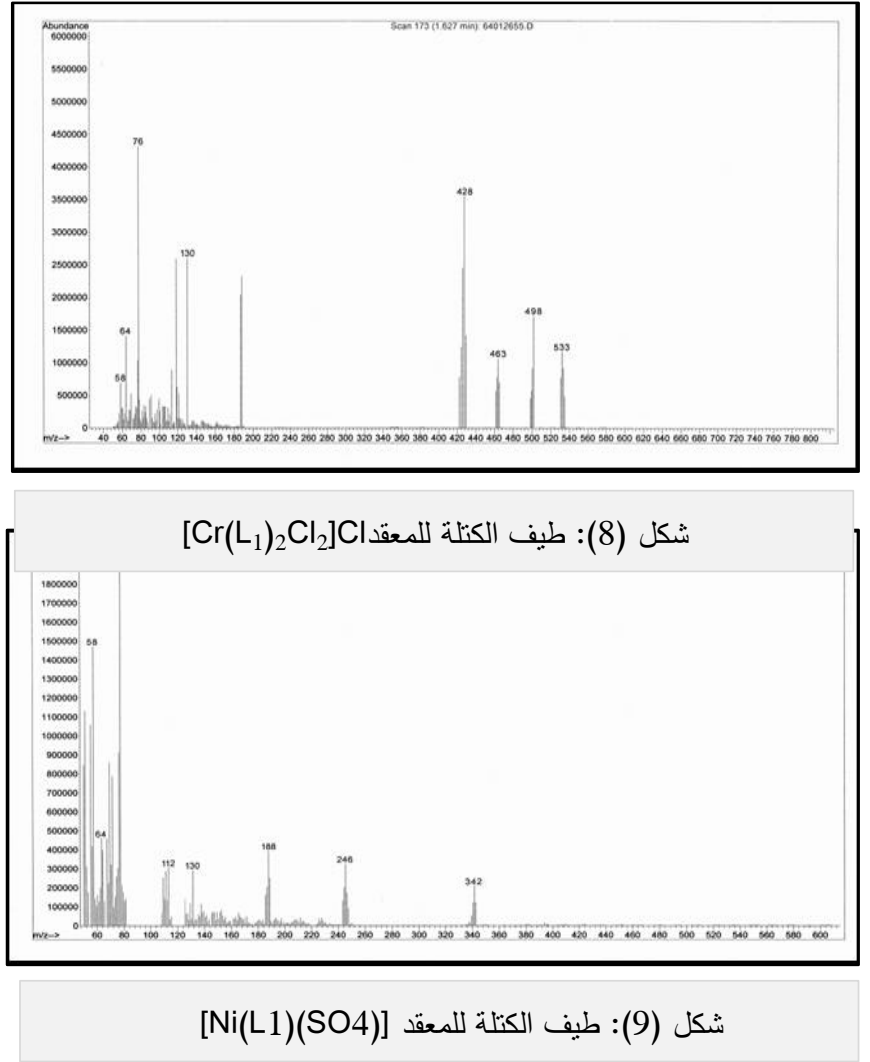

References

المصادر:

1- H. S. Al - Anssary, MSc. Thesis, Univ. of Basrah, (2011).

2- H. Schiff , Ann , 131 (1864) Citedim , R. W. Layer , Chem. Rev.Vol (63),pp. 489 , (1963).

3-Raman,N.andDhaveethu Raja, A series of copper complexes of mixed ligands with Schiff bases. Indian J. of chem. ,Vol (46)pp1611-1614 (2007).

4- Mohamed G G .synthesis, characterization and biological activity of bis(phenylamine) Schiff base liqands and their metal complezes,Spectrochim. Acta A Vol(64),pp 188 (2006).

5- M.R. Maurya, S.J.J. Titinchi and S.Chand ;"J.Mol. Catal. (A). Chem.;" Vol(193),pp165- 176, (2003).

6- Suresh, M.S and Prakash, International J. of Current Research, Vol 3, pp 3 (2011).

7- Kavita Rathore ,Rajivkr.Singh and H.B.Singh*EJ.of Chem.,( 2010).

8- Al.Mukhtar and I. A- Mustafa, "Inorganic and coordination chemistry ", Arabic version, Univ.of Mousl, (1988). 